\title{
LA IMPORTANCIA DE LAS TÉCNICAS DE TOMA DE NOTAS PARA LOS ESTUDIANTES DE INTERPRETACIÓN
}

\author{
Isidro José Fierro Ulloa ${ }^{1}$ \\ María Tatiana Hinojosa Navarrete ${ }^{2}$
}

\begin{abstract}
RESUMEN
Esta investigación es de carácter orientativo y pretende ayudar a los intérpretes a que adquieran un conocimiento general acerca de las técnicas para la toma de notas. El trabajo basa su problemática en el hecho de que en la asignatura de Interpretación no se ha establecido como prioritaria la enseñanza de técnicas en la toma de notas. Este artículo propone implementarlas en la malla curricular para mejorar las competencias de los estudiantes. Primero se hace un diagnóstico del nivel de los estudiantes y egresados de la carrera de Traducción e Interpretación; luego se realiza una revisión bibliográfica para determinar las técnicas existentes, de las cuales se propone finalmente la técnica de Jean-François Rozan. El marco teórico expone conceptos de la interpretación consecutiva, la toma de notas y las técnicas. La metodología utiliza el instrumento de la entrevista a una muestra de 15 estudiantes y 15 egresados de la Carrera de Traducción e Interpretación. Los resultados arrojados muestran la necesidad de que los estudiantes apliquen una o varias técnicas con el fin de optimizar su trabajo, dada la complejidad del proceso interpretativo y los diferentes retos que ha de afrontar a la hora de desempeñar un trabajo.
\end{abstract}

\section{PALABRAS CLAVES}

Interpretación, interpretación consecutiva, interpretación simultánea, toma de notas, técnica de Rozan.

\begin{abstract}
This research is instructive and aims to help the interpreters to attain general knowledge about the note taking techniques. This paper is based on the fact that in the translation classes, the teaching of note taking techniques has not been a priority. This article suggests its inclusion in the syllabus in order to improve the competences of the students. First off, we need to make a diagnosis of the translation and interpretation's students and alumni's level; after a bibliographic revision was made, we determined the existing techniques from which we finally propose Jean Francois Rozan's technique. The theoretical framework presents concepts of the consecutive interpretation, note taking and techniques. The methodology uses the survey to 15 students and 15 alumni as an instrument from the translation and interpretation program. The results of this research show that the students need to use one or several techniques in order to optimize their work due to the complexity of the translation process and the different challenges they have to tackle when it comes to do their job.
\end{abstract}

\section{KEYWORDS}

Interpretation, Consecutive Interpretation, Simultaneous Interpretation, Note Taking, Rozan's Technique.

Depositado en agosto 15 de 2014, aprobado en octubre 31 de 2014.

Investigación realizada como requisito para optar por el título profesional en Licenciatura en Traducción e Interpretación.

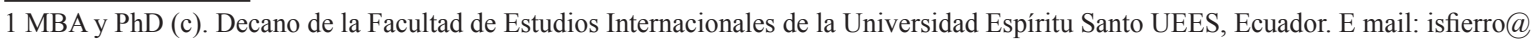
uess.edu.ec

2 Licenciada en Traducción e Interpretación de la Universidad Espíritu Santo UEES, Ecuador. Email: tatianahinojosa@gmail.com. 


\section{INTRODUCCIÓN}

Los intérpretes tienen que hacer en la actualidad frente a una serie de desafíos que requiere de un aprendizaje actualizado y competitivo. Entre los problemas que se encuentran, está el manejo de los nervios, la gestión del tiempo disponible para interpretar en la lengua de llegada, la capacidad de retención de la extensa información a interpretar, entre muchos otros. Estos problemas podrían ser factores que limiten al intérprete a la hora de entregar información precisa y completa.

La capacidad de análisis y de síntesis son fundamentales por la naturaleza propia de la interpretación y por las condiciones de trabajo del intérprete; generalmente se contrata a un intérprete poco tiempo antes de la celebración de la conferencia o del congreso, con lo cual éste dispone de un tiempo muy limitado para realizar su trabajo de documentación sobre el tema de que va a tratar la conferencia o el congreso. La capacidad de análisis y de síntesis son unas cualidades imprescindibles en el intérprete cuando debe asimilar, en poco tiempo, unos conocimientos básicos sobre un nuevo tema así como su terminología, e interpretar el discurso de manera fidedigna (Valdivia Campos, 1995)

Por fortuna existe la toma de notas, importante recurso en la interpretación consecutiva, destinada únicamente al uso inmediato del intérprete como un suplemento para la eficiencia de la memoria. Este recurso permite anotar información difícil de memorizar, sobre todo en la interpretación consecutiva, como largos discursos que contengan números, fechas, nombres, acrónimos, códigos, etc.

Para mejorar este recurso, existe una variedad de técnicas que contienen conceptos valiosos para ser transmitidos a los alumnos de interpretación. La técnica de Rozan, por ejemplo, utiliza una simbología propia y basa su importancia en la facilidad de su diseño y rápida transmisión, pues el intérprete se va a enfocar más en entender el mensaje que en transcribirlo. La metodología va a ser llevada a cabo mediante el instrumento de la entrevista y se va a recopilar información de parte de expertos que han utilizado exitosamente esta técnica.

Se espera que con los resultados de la presente investigación, los intérpretes nutran sus conocimientos sobre las técnicas que mejorarán su desempeño y habilidades en la toma de notas $\mathrm{y}$, a la vez, que se sienta la importancia de agregar estas técnicas a la malla curricular de los estudiantes de interpretación por parte de las autoridades pertinentes.

El objetivo del presente trabajo de investigación es, por tanto, identificar desde el punto de vista teórico los retos que enfrenta el profesional en lo referente a la toma de notas en la interpretación consecutiva. También se ofrece una perspectiva práctica, ya que se busca dar solución a unos problemas específicos de la interpretación mediante la propuesta de diferentes técnicas para mejorar las habilidades de la toma de notas. Finalmente, hay un aspecto metodológico, ya que existen varios métodos que el estudiante/ intérprete puede aplicar para hacer su trabajo más efectivo. Al desarrollar la técnica permitirá al estudiante una mayor competencia.

Se pretende que el estudiante mejore su aspecto curricular al incorporar todas las técnicas necesarias para facilitar o mejorar las habilidades y destrezas interpretativas mediante la eficiente toma de notas.

El estudio ha evidenciado que los egresados de los centros superiores en el área de la interpretación expresan que el profesional debe ampliar los conocimientos teóricos y prácticos sobre la toma de notas para así enfocarse mucho más en la comprensión del mensaje que se recepta del orador.

La información que se obtenga de este trabajo investigativo pretende mostrar la importancia de incluir en la malla curricular clases dedicadas a la enseñanza de las diferentes técnicas para la toma de notas.

\section{Revisión Literaria/Marco teórico}

Primero es necesario definir contextualmente el término "interpretación" con el propósito de 
obtener un conocimiento adecuado, aun cuando esta palabra tenga diferentes puntos de vista en cuanto a su significado. (Shuttleworth \& Cowie, 1997), por ejemplo, la definen en pocas palabras como "la traducción oral de un texto escrito". Hoy en día, se considera, además, que "la interpretación es la herramienta que permite la comunicación intercultural y la transmisión de información a nivel mundial" (VÁZQUEZ Y DEL ÁRBOL, 2005).

Existen dos tipos de interpretación: la modalidad consecutiva y la simultánea. "Comparado con la interpretación simultánea, la interpretación consecutiva se usa con más frecuencia" (Jin , 2010). El problema sobre decidir cuál modalidad utilizar es importante ya que se busca la interpretación más exacta en una forma que funcione efectivamente para todos los participantes en el evento. A continuación se definen ambas modalidades con base en las características del contexto planteado por diferentes situaciones interpretativas.

\section{(1) Interpretación Consecutiva -} modalidad en la cual el intérprete comienza la traducción oral del mensaje completo del discurso generado por el orador. El intérprete es la única persona en el contexto comunicacional que produce un mensaje en el momento en el que se realiza la interpretación. La interpretación consecutiva requiere de una buena memoria a corto plazo; algunos intérpretes incluso toman notas, aunque la mayoría confían simplemente en su memoria, en especial para expresiones cortas.

(2)InterpretaciónSimultánea-modalidad en la cual el intérprete y el orador hablan al mismo tiempo; el intérprete hace su traducción oral mientras recibe la expresión fuente. El intérprete no espera a que la idea completa haya sido expresada para hacer la traducción oral, pero termina unos segundos después de captar la idea completa del orador (Thuy Duong, 2006).

En la modalidad consecutiva de la interpretación, el orador realiza pausas en su discurso para dar tiempo suficiente a que el profesional haga su interpretación en la lengua de llegada. Existe una serie de ventajas cuando se elige esta modalidad: la consecutiva es más rentable, debido a que no requiere de equipos, se necesitan menos intérpretes y los participantes podrán escuchar ambas versiones de la interpretación (en la lengua de partida y de llegada). La ventaja para el intérprete es que tiene tiempo para asimilar la información del orador, reflexionarla, tomar notas (habilidad imprescindible) para trasmitir un mensaje fiel al original, etc.

En términos generales, esta habilidad se define como una técnica de simbolización gráfica por medio de la cual el intérprete registra, en paralelo a las operaciones de memorización, signos, términos o palabras que le permiten conservar -con vistas a la reformulación- aspectos lingüísticos e informativos del discurso. (Abuín González, 2004).

El propósito de la toma de notas en la interpretación es solo para el uso inmediato de la memoria del profesional. La toma de notas tiene como finalidad incrementar la eficiencia de la memoria, mas no escribir el discurso completo del orador. "De acuerdo con el modelo de esfuerzos de Gile para la interpretación consecutiva, el componente más peligroso que podría amenazar el equilibrio de la distribución de la energía mental es la toma de notas" (Meifang, 2012) . El exceso de la toma de notas, lo cual se debe evitar, durante la interpretación puede reducir la eficiencia de la memoria en vez de aumentarla. Se debe de tener en cuenta que la memoria es la que juega un papel principal en asegurar precisión en la interpretación y no la toma de notas, la cual es un sistema de apoyo para la memoria.

Aunque la toma de notas es bastante necesaria para la interpretación consecutiva, la paradoja puede ser que también es considerada como un "mal", debido a que puede dividir el poder de la atención e interferir en la escucha. La toma de notas es un trabajo frustrante, en especial para los principiantes de la interpretación; sin embargo, las buenas 
habilidades se pueden desarrollar a través de la suficiente práctica con la ayuda de los principios relativos a la toma de notas en la interpretación ( $\mathrm{Lu}, 2013)$.

Los futuros intérpretes profesionales deben aprender de las ideas y experiencias de los intérpretes experimentados que han hecho investigaciones exhaustivas sobre el tema para formar su propio sistema de notas. "la formación estratégica para la toma de notas en la profesión de la interpretación es ampliamente considerada como una táctica esencial de acoplamiento" (Chuang, s/f). Esto les permitirá estar listos para ofrecer un trabajo de interpretación con un nivel de alta calidad, y es por esto que el manejo de técnicas de toma de notas es indispensable.

Según Hong (Hong, 2006), "la toma de notas es una técnica útil para el trabajo del intérprete, sobre todo el que realiza la modalidad consecutiva". "En realidad, los estudiantes de interpretación aprenden el sistema de Rozan y luego desarrollan su propio método de toma de notas, compuesto de símbolos, abreviaturas y palabras en el idioma A, B, C o de una mezclas de estos" (Baselli, 2012).

Si bien no existe un solo método establecido sobre la toma de notas, hay varios principios fundamentales y métodos como los de Rozan, Gillies, Seleskovitch o Matyssek que brindan enfoques teóricos sobre esta habilidad. Al ser un sistema personalizado, no se cuenta con una teoría específica al respecto, pero estos métodos proponen ejercicios para que cada estudiante de interpretación practique y pueda desarrollar su propio método. Con este propósito, a continuación se expondrá el método de Rozan y sus principios útiles para mejorar esta destreza.

\section{El manual de Jean-François Rozan}

El uso de una técnica depende por lo general de la aplicación de un número de principios; esto es lo que llamamos instrucciones. Uno no tiene que seguir las reglas recomendadas en dichas instrucciones; de hecho, el producto, dispositivo o sistema para el que fueron creados pueden funcionar o trabajar incluso si no se observa, pero lo harán con menos eficiencia. Por otra parte, mientras más simples sean las instrucciones, existe más probabilidad de que el usuario las siga. Esta misma situación se aplica en la toma de notas. Unos principios muy simples dan a este método o conjunto de principios gran precisión y facilitan su aplicación por parte del intérprete (Waliczek \& Gillies, 2002).

El concepto que Jean-François Rozan plantea sobre la toma de notas, es un método sencillo y minimalista basado en siete principios y 20 símbolos, que contiene elementos valiosos para ser transmitidos a los alumnos. Un método factible es el de empezar con los principios básicos de Rozan, ya que pone en conjunto algunas ideas para ayudar a los interpretes con la tarea de la toma de notas.

\section{Los siete principios de Jean-François Rozan}

1. Darle prioridad a la idea antes que a la palabra.

El primer principio se enfoca en el sentido, el cual es más importante que las palabras. En el momento de tomar notas se debe tener en cuenta las ideas que el discurso pretende transmitir. Usualmente se utilizan ciertas abreviaturas y símbolos con los que el profesional debió previamente haberse familiarizado. Es muy importante tener presente que el proceso de lectura de notas no debería ser sinónimo de un proceso de descifrado, esto quiere decir que no se debe abusar de los símbolos. En resumen, analizar el discurso y posteriormente anotar la idea principal y sus ideas secundarias.

2. Las reglas de abreviatura.

Rozan expresa que la mejor forma de abreviar palabras largas es usando de ellas las primeras y las ultimas letras, esto es para no caer en confusiones con palabras similares que contengan terminaciones diferentes.

Por ejemplo: production $=\operatorname{Pr}^{\text {on }}$

3. Conectores.

Según el autor de estos principios, en el también llamado encadenamiento es indispensable apuntar las diferentes relaciones lógicas establecidas entre las ideas del discurso; los conectores ayudan a reformular las ideas.

Por ejemplo: $b u t=$ pero, $\sin$ embargo, 
aunque, no obstante, etc.

4. Negación.

Se realiza un trazo oblicuo o se tacha la palabra o símbolo a la que se le quiera dar un sentido de prohibición, negación u oposición.

5. Énfasis.

El énfasis o acentuación se refiere al hecho de subrayar una o dos veces a la palabra para indicar su importancia.

6. Verticalidad.

Este principio le brinda más elegancia a la interpretación, debido a que permite recoger de un vistazo todas las ideas del discurso. Rozan recomienda una toma de notas estructurada de forma vertical.

7. Escalonamiento.

Este último principio se trata de los espaciados, y consiste en posicionar los símbolos o palabras en las líneas inferiores, para que, de esta manera, formen una unidad con los elementos que se encuentran en la línea superior a las que se refieren sin que exista la necesidad de repetir estos elementos.

Jean-François Rozan además propone 20 símbolos:

1. De expresión.

Símbolos de la palabra "

Símbolo del pensamiento :

Símbolo del debate

Símbolo de la aprobación $\mathrm{OK}$

2. De movimiento.

Flecha de orientación

Flecha de aumento

Flecha de disminución

3. De correspondencia.

Símbolo de relación

Símbolo de igualdad

Símbolo de diferencia $\quad \neq$

Símbolo de encadenamiento

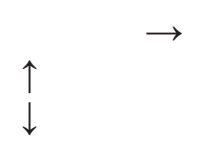

Símbolo positivo +

Símbolo negativo -

4. Substantivos.

De país

Internacional, extranjero $\nabla \nabla^{\prime} \nabla^{\times}$

Mundial, universal, global W (world)

Trabajo, acción, labor (work)

Cuestión, problema, aspecto
Miembro, participante, delegado MS $\triangle$ Comercio, relaciones comerciales TR $\rightleftarrows$ (trade)

El desarrollo de la habilidad de la toma de notas es de importancia estratégica para lograr una exitosa interpretación consecutiva. El propósito de las notas, en la interpretación, es que sirven como refuerzo a la memoria, proporcionan pistas visuales y dan acceso a elementos difíciles de memorizar en una interpretación de larga duración. Sin el desarrollo o utilización de un sistema de notas minimalista, la codificación y decodificación de los apuntes tenderán a complicar el proceso interpretativo (Chuang, $\mathrm{s} / \mathrm{f})$. Afortunadamente, existe una variedad de técnicas, como la de Rozan, que ayudan a que el estudiante de interpretación o el intérprete aprenda de éstas para posteriormente crear su propio sistema de carácter simple y economizador.

Debido a la evidente importancia de las técnicas en la formación de los intérpretes, se considera vital que se agregue a la malla curricular de los estudiantes la enseñanza-aprendizaje de los diferentes métodos existentes en el mercado para ayudar al individuo a mejorar su capacidad interpretativa.

\section{Formulación del problema}

La interpretación, como el Sudoku, es un "juego" que requiere de destreza mental, sentido crítico, buena memoria, retentiva, disciplina mental, capacidad de analizar y resolver problemas, e intuición. Cada "juego" trae nuevos desafíos y es diferente, ya que hace que el intérprete adquiera más experiencia y conocimiento. Lo que hace a un buen intérprete es la disciplina empleada en sus estudios, numerosos ejercicios de práctica, además de las técnicas y habilidades adquiridas; e, indudablemente, una de las habilidades que el estudiante de interpretación debe pulir y dominar es la toma de notas.

La toma de notas en la interpretación consecutiva es una habilidad que se debe aprender. No existe una teoría abstracta acerca de esta habilidad, pero hay una amplia gama de principios funcionales 
establecidos por las sucesivas generaciones de intérpretes consecutivos a lo largo de la historia. Estos principios se han hecho de los dos estudios empíricos que cavan a profundidad en casi todos los aspectos de la habilidad y de los libros de investigación que elaboran las principales aproximaciones teóricas (Hong, 2006).

Como estudiante de la Facultad de Estudios Internacionales, a la autora siempre le llamó la atención que en el campo de la interpretación consecutiva no se apliquen técnicas específicas para la toma de notas. Esta actividad se ha venido realizando en forma empírica y depende absolutamente del estudiante el encontrar rapidez y seguridad en un marco científicopráctico, pues es conocido que una técnica sólida facilita el desarrollo a quienes se desempeñan como intérpretes. El dominio de varias técnicas ayudará a los jóvenes universitarios de hoy a cursar la asignatura de interpretación como parte de su formación, que será de mucha utilidad para su entrada al mundo laboral. Tal como lo expresa (Opdenhoff, 2004): "una buena técnica de toma de notas es imprescindible".

La problemática se genera porque en la malla curricular de la carrera de Interpretación no se considera la enseñanza de las técnicas que se requieren para mejorar las competencias de los estudiantes y egresados en las actividades de toma de notas. Hay que tener en cuenta que la memoria cumple la función principal en el papel del intérprete y que, para que su desempeño sea bueno, debe manejar las técnicas de toma de notas que le permitan enfocarse en entender el mensaje y no solo en anotar lo que se dice.

\section{Metodología}

El presente paper obedece a los lineamientos de la investigación descriptiva, documental y explicativa. Es de carácter descriptivo, ya que identifica y detalla las características fundamentales del objeto de estudio: la toma de notas y la relevancia de la enseñanza-aprendizaje de las técnicas para mejorar esta habilidad. Por tanto, brinda información necesaria para que el intérprete aplique los aspectos técnicos en su vida laboral. La investigación estará respaldada con los resultados que se obtengan de las encuestas realizadas a los estudiantes y ex estudiantes de la carrera de traducción e interpretación. Se considera de tipo documental debido a que se ha acudido a información existente sobre el tema que se decidió examinar. Existen libros, artículos de libros, revistas, periódicos, y más recursos relacionados con el tema. En este proyecto se aplica, además, la modalidad de proyecto factible. El estudio se ha hecho en el entorno de los estudiantes del Instituto de Educación Superior y con los egresados que se están desempeñando en diferentes cargos de su especialización. Debido a que, como se mencionó anteriormente, se trabajará con el total de la población, la encuesta es el método apropiado para recolectar datos. Este instrumento permitirá recopilar datos sobre sucesos anteriores a los entrevistados y permitirá estandarizar la información para posteriormente realizar un análisis. La observación participante es otra herramienta útil en esta investigación, ya que el investigador y responsable de recolectar los datos se involucra directamente con la actividad objeto de la observación.

Todo esto está de acuerdo con Meyer \& Van Dalen (1978), cuando manifiestan que:

\section{El objetivo de la investigación} descriptiva consiste en llegar a conocer las situaciones, costumbres y actitudes predominantes a través de la descripción exacta de las actividades, objetos, procesos y personas. Su meta no se limita a la recolección de datos, sino a la predicción e identificación de las relaciones que existen entre dos o más variables.

\section{Investigación de campo}

Nerici Imideo (1968) en su libro Hacia una didáctica general dinámica define así la investigación de campo:

Es el estudio sistemático de problemas, en el lugar en que se producen los acontecimientos con el propósito de descubrir, explicar sus causas y efectos, entender su naturaleza e implicaciones, 
establecer los factores que lo motivan y permiten predecir su concurrencia (p. 32).

La investigación aquí presentada se realizó en la Institución de Educación Superior para la Carrera de Traducción e Interpretación en la ciudad de Guayaquil, Ecuador.

\section{Población y muestra}

La población utilizada para desarrollar este trabajo investigativo la componen 15 estudiantes y 15 egresados de la carrera de Traducción e Interpretación de la UEES, relacionados todos con los años que ha durado esta carrera hasta el cierre de la misma. Dado que la población es limitada (menos de 30 personas), no es necesario utilizar la fórmula de proporciones en esta investigación.

\section{Análisis de los resultados}

Encuesta a estudiantes y egresados 1.- ¿Considera usted, como estudiante y como egresado, que para desarrollar de manera eficiente, rápida y objetiva su tarea de interpretación mediante la toma de notas, debe dominar algunas técnicas que le faciliten esta tarea?

Gráfico 1:

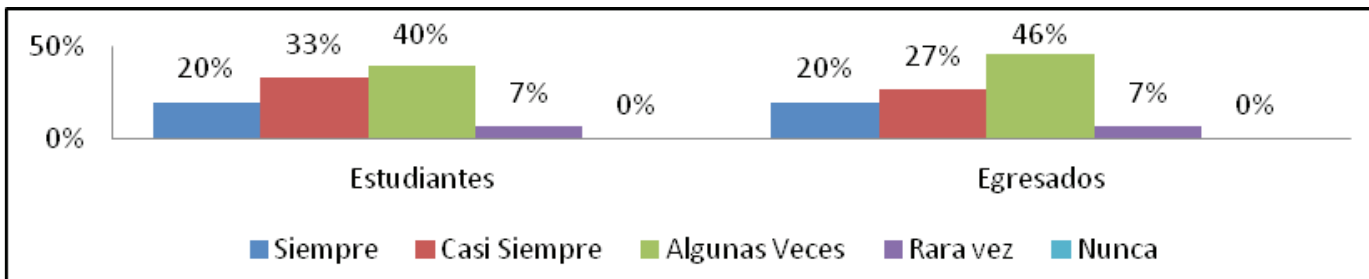

Fuente: Tabulación de encuesta a estudiantes y egresados, Carrera Traducción e Interpretación. Elaborado por: María Tatiana Hinojosa Navarrete.

Análisis:

Estudiantes y egresados consideran que, algunas veces, para desarrollar de manera eficiente, rápida y objetiva su tarea de interpretación mediante la toma de notas, debe dominar algunas técnicas que le faciliten esta tarea.

2.- ¿Las técnicas como herramientas para facilitar la enseñanza aprendizaje de la interpretación mediante toma de notas fueron incluidas en el pénsum?

Gráfico 2:

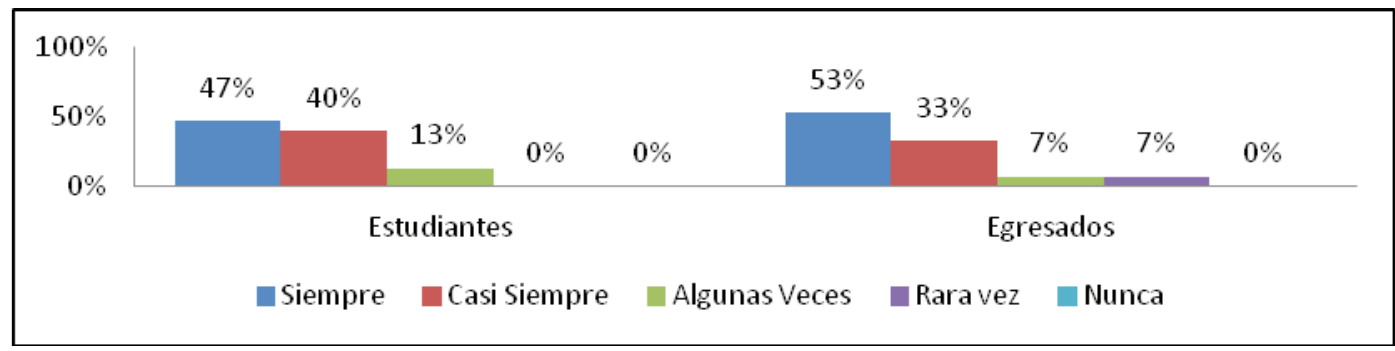

Fuente: Tabulación de encuesta a estudiantes y egresados. Carrera Traducción e Interpretación.

Elaborado por: María Tatiana Hinojosa Navarrete. 


\section{Análisis:}

Los resultados de la encuesta señalan que las técnicas como herramientas para facilitar la enseñanza aprendizaje de la interpretación mediante toma de notas no fueron incluidas en el pénsum.

3.- ¿Usted considera que dentro de la enseñanza integral de la especialización en interpretación, la autoridad de la Institución superior deberá incluir en la malla curricular los materiales de técnicas para mejorar la habilidad del estudiante en la toma de notas?

\section{Gráfico 3:}

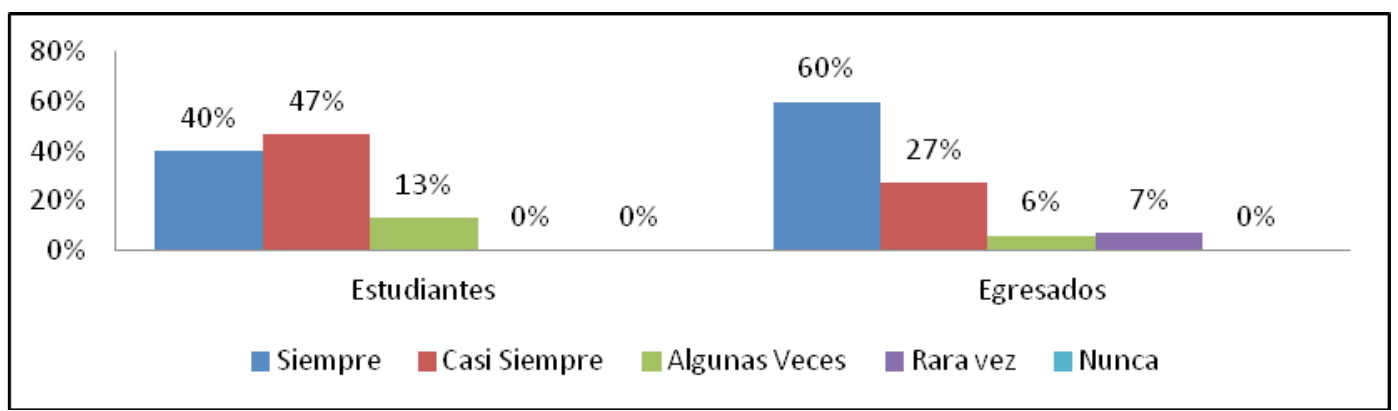

Fuente: Tabulación de encuesta a estudiantes y egresados. Carrera de Traducción e Interpretación.

Elaborado por: María Tatiana Hinojosa Navarrete.

\section{Análisis:}

Los resultados obtenidos de la encuesta dirigida a los integrantes de esta investigación, es decir, estudiantes y egresados, expresan que están de acuerdo en que la autoridad incluya en la malla curricular materiales sobre técnicas para mejorar la habilidad del estudiante en la toma de notas.

4.- ¿Está dispuesto a capacitarse en técnicas destinadas a mejorar los componentes de interpretación mediante la toma de notas?

Gráfico 4:

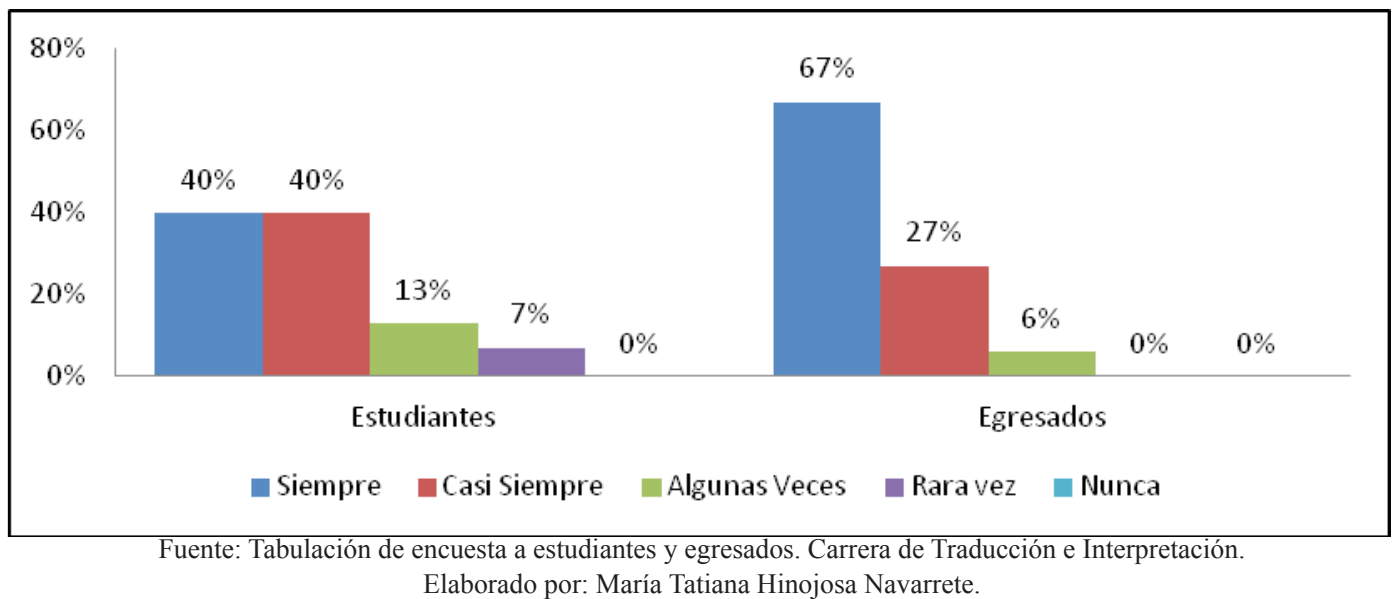

\section{Análisis:}

Los resultados obtenidos de la encuesta realizada a los estudiantes y egresados en la carrera de Traducción e Interpretación, señalan siempre que éstos están dispuestos a capacitarse en técnicas 
destinadas a mejorar los componente de interpretación mediante la toma de notas. Consideran que el pénsum de la enseñanza que deben recibir de sus respectivas escuelas, no sólo debe ser de carácter general sino de especialización; de esta manera, la percepción en el pronóstico del desempeño profesional será más eficiente y de un reconocimiento laboral que se transforme en una mejor remuneración económica.

5.- ¿En su percepción, las autoridades del plantel están dispuestas a incorporar en la malla curricular la asignatura de técnicas y herramientas para facilitar la tarea del estudiante y del profesional de la interpretación mediante la toma de notas?

\section{Gráfico 5:}

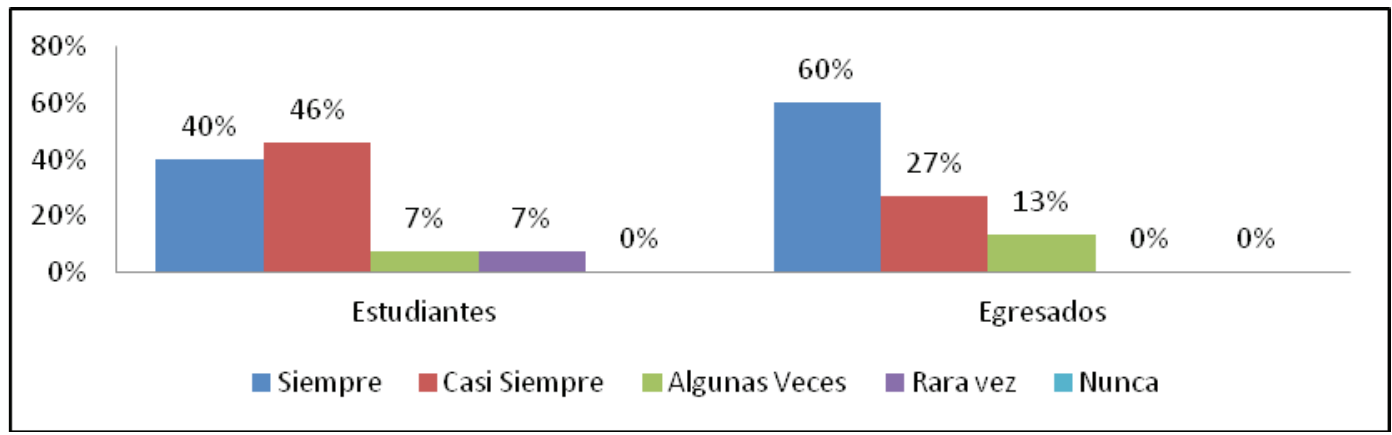

Fuente: Tabulación de encuesta a estudiantes y egresados. Carrera de Traducción e Interpretación. Elaborado por: María Tatiana Hinojosa Navarrete.

\section{Análisis:}

La encuesta realizada a los estudiantes y egresados de la carrera de Traducción e Interpretación, da como resultado que una mayoría de cada uno de los grupos considerados en la presente investigación resalta la necesidad de que en el pénsum de la carrera, además de las técnicas que usualmente se enseñan en el aula de clase, se incorpore la técnica de Rozan. De acuerdo con la opinión recogida se interpreta que a criterio de los consultados la técnica de Rozan debe ser tenida en cuenta en la enseñanza, en razón de reunir condiciones generales y específicas que la convierten en una herramienta clave para fortalecer la labor del profesional que ejercita la toma de notas.

6.- ¿Siendo la técnica de Rozan una herramienta básica que no ha perdido actualidad debe ser incluida en la malla curricular?

\section{Gráfico 6:}

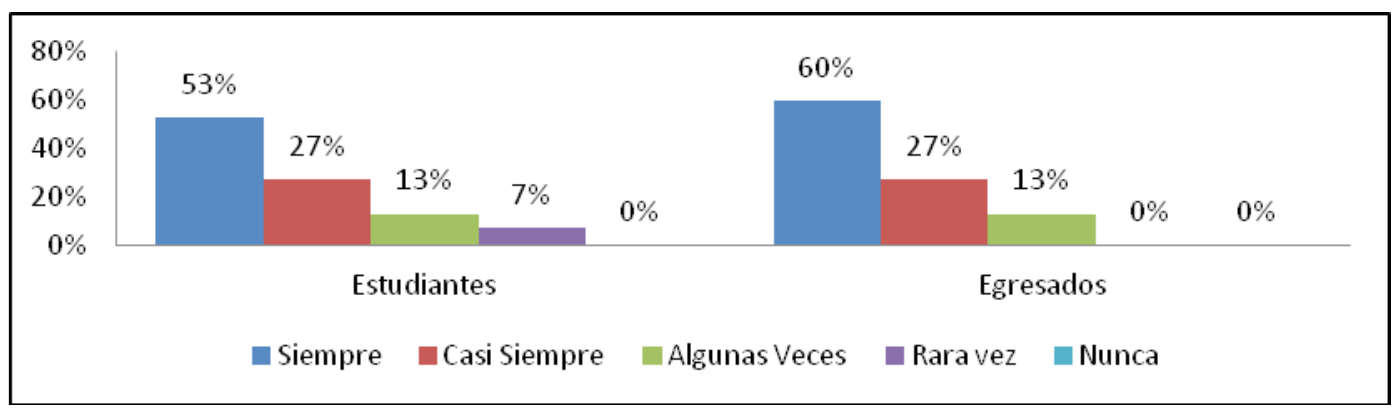

Fuente: Tabulación de encuesta a estudiantes y egresados. Carrera de Traducción e Interpretación. Elaborado por: María Tatiana Hinojosa Navarrete. 


\section{Análisis:}

La encuesta aplicada a los estudiantes y egresados de la carrera de Traducción e Interpretación permite conocer que la mayoría de ellos está de acuerdo en que el desempeño laboral no debe estar condicionado al dominio de una sola técnica; todo lo contrario, es imprescindible que el profesional cuente en su labor académica con un conjunto de técnicas que le den mayor solvencia y eficiencia en la toma de nota para generar una interpretación fidedigna a la fuente de entrada, de tal manera que su tarea sea el reflejo exacto o más aproximado de lo expresado por el emisor.

\section{Interpretación de los resultados}

La encuesta recoge la opinión de los estudiantes egresados de la carrera de Traducción e Interpretación, la cual está expresada en los siguientes términos. En ambos sectores, la mayoría de encuestados señala que la técnica de Rozan les fue requerida en muchos casos, pero que, lamentablemente, por no conocerla y dominarla, no la pudieron aplicar. Lo anterior sugiere a las autoridades de los centros superiores que enseñan esta especialidad la necesidad de estudiar más a fondo estas técnicas para establecer la posibilidad de incorporarlas en el pénsum de la enseñanza.

Los resultados permiten el siguiente comentario interpretativo: tanto los estudiantes como los egresados de la Escuela de Traducción e Interpretación muestran el convencimiento de que técnicas como la de Rozan deben estar incluidas en el pénsum de la enseñanza, y que los Organismos de Educación Superior deben buscar la manera de atender a este pedido. De darse este paso, el beneficiado sería el estudiante, ya que su pénsum será tomado en cuenta para resolver el pedido de los encuestados.

Tanto los estudiantes como los egresados, en un porcentaje importante de la población encuestada, coinciden en que se deben realizar tareas de evaluación de su desempeño en cuanto a la aplicación de las técnicas, con el objeto de obtener un diagnóstico que permita determinar sus limitaciones o virtudes en el manejo de dichas herramientas e incentivar la adquisición de otras nuevas mediante la capacitación correspondiente.

\section{Conclusiones}

Se ha determinado que los estudiantes consideran que el conocimiento empírico para desarrollar la toma de notas en la actividad de la interpretación del mensaje de salida no es suficiente, sobre todo, porque en la práctica queda claro que, de acuerdo con el idioma y el contexto en que se realiza la toma de notas, el profesional de la interpretación debe saber manejar varias técnicas y, entre ellas, elegir la que más convenga.

La carrera de Traducción e Interpretación no ha implementado en su currículum las diversas técnicas existentes; por tal motivo, los estudiantes y egresados desconocen que el ejercicio de la profesión impone una variedad amplia de técnicas que han de ajustarse a las distintas circunstancias o exigencias que el cliente demanda.

\section{Recomendaciones}

Las autoridades de la carrera deberían recoger la opinión de los estudiantes y egresados, en el sentido de promover una formación académica de la toma de notas basada en la enseñanzaaprendizaje de todas las técnicas existentes en este campo con el objeto de elevar el currículum educativo.

Los Centros Superiores del país deben incluir en su Plan Educativo Institucional las diferentes técnicas desarrolladas en el campo de la investigación, con el fin de dotar a estos sectores socio-educativos de un currículo amplio que garantice que la toma de nota sea más eficiente, rápida y objetiva. 


\section{Referencias bibliográficas}

Abuín González, M. (2004). Problemas y estrategias en la interpretación consecutiva: consideraciones metodológicas. Madrid : Universidad de la Comunidad de Madrid.

Baselli, V. (03 de Diciembre de 2012). Which language do interpreters use in Consecutive Interpreting? Proceedings in ARSA-Advanced Research in Scientific Areas, 1. Milan, Italy.

Chuang, L.-1. (s/f). Note-taking Know-how:A Processing Perspective on Consecutive Interpreting. Spectrum: Studies in Language, Literature, Translation, and Interpretation, 2, 93-101.

Hong, P. (2006). Note-taking in Consecutive Interpreting . Obtenido de https://www.google.com.ec/url? sa=t\&rct=j\&q=\&e $\mathrm{src}=\mathrm{s} \&$ source $=$ web \&cd $=1 \& \mathrm{cad}=\mathrm{rja} \& \mathrm{ved}=0 \mathrm{CCcQFjAA} \& u r l=\mathrm{http} \% 3 \mathrm{~A} \% 2 \mathrm{~F} \% 2 \mathrm{Fweb}$. hanu.vn $\% 2 \mathrm{Fen} \% 2 \mathrm{Ffile} . \mathrm{php} \% 2 \mathrm{~F} 1 \% 2$ Fmoddata\%2Fforum\%2F70\%2F393\%2FNOTE-TAKING_IN_CONSECUTIVE_INTERPRETING_pdf\&ei=LnhVUpaK IYy89QS6nIGwCw\&usg=AFQjCNGqiRA

Jin , Y. (2010). T21N - Translation in Transition: The Conceptual Mapping Model in Consecutive Interpreting Teaching. (W. V. Trier, Ed.) Auckland.

Lu, W. (2013). Developing note-taking skills in consecutive interpreting. En Linguistics, Culture \& Education (Vol. 2, págs. 72-81).

Meifang, Z. (2012). The Study of Note-taking and Memory in Consecutive Interpretation. Nanjing: Tianhe College of Guangdong Polytechnic Normal University. Obtenido de http://www.ier-institute.org/2070-1918/lnit16/v16/178.pdf

Meyer, W., \& Van Dalen, D. (1978). Manual de técnica de la investigación educacional. Editorial Paidós.

Nerici, I. (1968). Hacia una didáctica general dinámica. Buenos Aires: Kapelusz.

Opdenhoff, J.-H. (2004). Alfa, épsilon, pi-Algunas reflexiones sobre la toma de notas en la enseñanza de la interpretación consecutiva. TRANS: revista de traductología 8, 161-168.

Shuttleworth , M., \& Cowie , M. (1997). Dictionary of Translation Studies. New York: St. Jerome Publishing .

Thuy Duong, T. (2006). How to improve short-term memory in interpreting .

Valdivia Campos , C. (1995). La interpretación. Anales de filología francesa, 7, 175-181.

VÁZQUEZ Y DEL ÁRBOL, E. (2005). Herramientas metodológicas para la formación de intérpretes: el modo simultáneo. En M. Romana García (Ed.), Actas del II Congreso Internacional de la Asociación Ibérica de Estudios de Traducción e Interpretación. Madrid.

Waliczek, B., \& Gillies, A. (2002). Note-taking in consecutive interpreting. Krakow: Tertium. 
APÉNDICE

\section{CUESTIONARIO DIRIGIDO A ESTUDIANTES Y EGRESADOS DE LA CARRERA DE TRADUCCIÓN E INTERPRETACIÓN}
$5=$ Siempre
$2=$ Rara vez
$4=$ Casi siempre
$1=$ Nunca

$3=$ Algunas veces

\begin{tabular}{|c|c|c|c|c|c|c|}
\hline No. & Preguntas & 5 & 4 & 3 & 2 & 1 \\
\hline 1. & $\begin{array}{l}\text { ¿Considera usted, como estudiante y como egresado, que para desarrollar } \\
\text { de manera eficiente rápida y objetiva su tarea de interpretación mediante } \\
\text { la toma de notas, debe dominar algunas técnicas que le faciliten esta } \\
\text { tarea? }\end{array}$ & & & & & \\
\hline 2. & $\begin{array}{l}\text { ¿Las técnicas como herramientas para facilitar la enseñanza aprendizaje } \\
\text { de la interpretación mediante toma de notas fueron incluidas en el } \\
\text { pénsum? }\end{array}$ & & & & & \\
\hline 3. & $\begin{array}{l}\text { ¿Usted considera que dentro de la enseñanza integral de la especialización } \\
\text { en interpretación, la autoridad de la Institución superior deberá incluir en } \\
\text { la malla curricular los materiales de técnicas para mejorar la habilidad } \\
\text { del estudiante en la toma de notas? }\end{array}$ & & & & & \\
\hline 4. & $\begin{array}{l}\text { ¿Está dispuesto a capacitarse en técnicas destinadas a mejorar los } \\
\text { componentes de interpretación mediante la toma de notas? }\end{array}$ & & & & & \\
\hline 5. & $\begin{array}{l}\text { ¿En su percepción, las autoridades del plantel están dispuestas a } \\
\text { incorporar en la malla curricular la asignatura de técnicas y herramientas } \\
\text { para facilitar la tarea del estudiante y del profesional de la interpretación } \\
\text { mediante la toma de notas? }\end{array}$ & & & & & \\
\hline 6. & $\begin{array}{l}\text { ¿Siendo la técnica de Rozan una herramienta básica que no ha perdido } \\
\text { actualidad debe ser incluida en la malla curricular? }\end{array}$ & & & & & \\
\hline
\end{tabular}

